\begin{tabular}{c|c|c}
\hline ISSN 2525-4812 (versão online) & Revista Terceira \\
$\begin{array}{c}\text { ISSN 2238-7641 (versão impressa) } \\
\text { http://www.revistaterceiramargem.com/ } \\
\text { index.php/terceiramargem/index }\end{array}$ & $\begin{array}{c}\text { Recebido em: 19/6/2019 } \\
\text { Aprovado em: } 30 / 2 / 2020\end{array}$ & $\begin{array}{c}\text { Revisaço } \\
\text { Margem Amazônia }\end{array}$ \\
\hline
\end{tabular}

Como citar o artigo:

CARNEIRO, J, P. R; PEREIRA, C. F; SANTIAGO, J. L; FRAXE, T. J. P. Sustentabilidade e política públicas: Impactos do Programa de Agquisição de Alimentos - PAA na vida dos agricultores familiares em Rio Preto da Eva - AM. Revista Terceira Margem Amazônia, v. 6, n.15, p. 135-149, 2020. Doi: http://dx.doi.org/10.36882/2525-4812.2020v6i15p135-149.

\title{
SUSTENTABILIDADE E POLÍTICAS PÚBLICAS: IMPACTOS DO PROGRAMA DE AQUISIÇÃO DE ALIMENTOS - PAA NA VIDA DE AGRICULTORES FAMILIARES EM RIO PRETO DA EVA - AM
}

\author{
Janderlin Patrick Rodrigues Carneiro ${ }^{1}$ \\ Cloves Farias Pereira ${ }^{2}$ \\ Jozane Lima Santiago ${ }^{3}$ \\ Therezinha de Jesus Pinto Fraxe 4
}

\begin{abstract}
Resumo: Este artigo tem como objetivo avaliar os efeitos gerados na sustentabilidade da agricultura familiar a partir da operacionalização do Programa de Aquisição de Alimentos - PAA. Buscando caracterizar os aspectos socioeconômicos e ambientais dos agricultores familiares e analisar as implicações do PAA na sustentabilidade da agricultura familiar. Para tanto, utilizou-se o estudo de caso, os resultados foram obtidos através de aplicação de formulários socioeconômicos, questionário sobre a frequência alimentar e aplicada a Escala Brasileira de Insegurança Alimentar - EBIA. A escala apontou a eficiência do PAA no seu contexto mais abrangente, abarcando desde suas origens, execuções, modalidades chegando até seu ápice, demonstrando como pode ser também um canal para construir a sustentabilidade. Esse entendimento, tornou-se essencial para traçar novos caminhos a partir dos resultados da pesquisa, em que evidencia aspectos importantes da sustentabilidade. Procurando compreender a sustentabilidade através da ótica das políticas públicas, sobretudo aquelas que promovem a importância da agricultura familiar. É relevante ressaltar que o fortalecimento de políticas voltadas para a agricultura
\end{abstract}

\footnotetext{
${ }^{1}$ Assistente Social, Mestre em Ciências do Ambiente e Sustentabilidade na Amazônia pela Universidade Federal do Amazonas - UFAM. Pesquisador do Núcleo de Socioeconomia da Universidade Federal do Amazonas, E-mail: patrickcarneiro09@gmail.com

${ }^{2}$ Doutor em Antropologia Social pela Universidade Federal do Amazonas. Professor Adjunto da Universidade Federal do Amazonas (UFAM). Pesquisador do Núcleo de Socioeconomia. E-mail: cloves.canarana@ gmail..com

${ }^{3}$ Doutora em Ciências do Ambiente e Sustentabilidade na Amazônia pela Universidade Federal do Amazonas. Professora Adjunta da Universidade Federal do Amazonas (UFAM). Vice-coordenadora do Núcleo de Socioeconomia. E-mail: jozaneagroecologia@gmail.com

${ }^{4}$ Doutora em Sociologia pela Universidade Federal do Ceará (UFC). Professora Titular da Universidade Federal do Amazonas (UFAM). Coordenadora do Núcleo de Socioeconomia. E-mail: tecafraxe@uol.com.br
} 
familiar mostra-se como estratégia de sustentabilidade, assim como a inserção da agricultura familiar como estratégia de desenvolvimento rural.

Palavras-chave: sustentabilidade; agricultura familiar; políticas públicas; paa

Abstract: This article aims to evaluate the effects generated on the sustainability of family farming from the operationalization of the Food Acquisition Program - PAA. Seeking to characterize the socioeconomic and environmental aspects of family farmers and analyze the implications of the PAA on the sustainability of family farming. For this, we used the case study, the results were obtained through the application of socioeconomic forms, food frequency questionnaire and the Brazilian Food Insecurity Scale - EBIA. In which, he pointed out the efficiency of the PAA in its broader context, covering from its origins, executions, modalities reaching its peak, demonstrating how it can also be a channel to build sustainability. This understanding has become essential to draw new paths from the research results, which highlights important aspects of sustainability. Seeking to understand sustainability through the perspective of public policies, especially those that promote the importance of family farming. It is important to emphasize that the strengthening of policies aimed at family farming is shown as a sustainability strategy, as well as the insertion of family farming as a rural development strategy.

Keywords: sustainability; family farming; public policy; paa

\section{INTRODUÇÃO}

Este trabalho irá abordar os resultados da dissertação de mestrado apresentado ao Programa de pós-graduação em Ciências do Ambiente e Sustentabilidade da Universidade Federal do Amazonas, intitulado "Impactos do Programa Aquisição de Alimentos - PAA na sustentabilidade de agricultores familiares no Município de Rio Preto da Eva - AM", o qual retrata de forma concreta os efeitos do Programa na vida dos agricultores familiares do ponto de vista da sustentabilidade.

Dessa forma, este trabalho mostra um comparativo entre agricultores familiares fornecedores e não fornecedores, chamamos agricultores fornecedores aqueles que negociam seus produtos com o PAA, enquanto os agricultores não-fornecedores negociam os seus produtos com atravessadores e/ou na cidade de Rio Preto da Eva. Neste sentido, foi possível comparar os dois grupos de agricultores familiares. Tal empreitada possibilitou avaliar os impactos gerados pelo PAA aos agricultores fornecedores quando comparado aos agricultores não-fornecedores.

Os sujeitos da pesquisa foram agricultores familiares associados a Associação dos Produtores Rurais da Comunidade Nova Esperança (ASPRONES), localizada no 
Município Rio Preto da Eva no Estado do Amazonas. O objetivo deste trabalho foi avaliar os efeitos gerados na sustentabilidade da agricultura familiar a partir da operacionalização do programa PAA. Buscando caracterizar os aspectos socioeconômicos e ambientais dos agricultores familiares e analisar as implicações do PAA na sustentabilidade da agricultura familiar. Para tanto, utilizou-se o estudo de caso, pois este possibilita ao pesquisador uma abordagem mais abrangente com a utilização de inúmeras técnicas, sendo possível reter características significativas dos eventos da vida real. (YIN, 2001, p. $32)$.

Os resultados foram obtidos através de aplicação de formulários socioeconômicos, questionário sobre a frequência alimentar e utilizada a Escala Brasileira de Insegurança Alimentar - EBIA., a partir da dimensão da sustentabilidade dos agricultores familiares. Portanto, apresenta didaticamente elementos que estabelecem análises dos impactos das ações governamentais na sustentabilidade dos agricultores familiares.

\section{PROGRAMA DE AQUISIÇÃO DE ALIMENTOS - PAA: FERRAMENTA ESTRATÉGICA PARA AGRICULTORES FAMILIARES.}

Criado em 2003, o programa surge como uma das principais ações estruturantes do Programa Fome Zero, com proposta de fortalecer agricultura familiar, auxiliando agricultores a ampliar seu acesso a novos mercados (MULEER; FIALHO e SCHNEIDER, 2007). Yazbek (2004) assegurou que o programa foi desenhado para oferecer alternativas de venda da produção, criando uma opção para geração e incremento de renda dos agricultores familiares. Confirma sua importância para a inclusão social no campo e valorização dos produtores locais (BECKER e SACCO DOS ANJOS, 2010).

Soares et al. (2013. p. 42) enfatizou que:

O Programa tem o objetivo de garantir, a populações em situação de insegurança alimentar e nutricional, o acesso a alimentos em quantidade, qualidade e regularidade necessárias; promover a inclusão social no campo por meio do fortalecimento da agricultura familiar; promover o abastecimento institucional com alimentos para diversos fins, como a alimentação escolar; e constituir estoques estratégicos.

Do ponto de vista legal, o PAA é instituído pela Lei $\mathrm{n}^{\circ} 10.696$, de 02 de julho de 2003, com finalidade de incentivar a agricultura familiar, compreendendo ações vinculadas à distribuição de produtos agropecuários para pessoas em situação de insegurança alimentar e à formação de estoques estratégicos. Regulamentado pelo 
Decreto n ${ }^{\circ} 7.775$, de 04 de maio de 2012, onde delibera sobre o público do programa, da aquisição e destinação de alimentos, das modalidades de execução, unidades gestoras, dentre outras.

Com esse arcabouço legal, o PAA ganha legitimidade no âmbito nacional, sendo instituído como política de Estado, não mais de governo. Para tanto, se faz necessário manter uma estruturação, que é realizada pelo grupo gestor do PAA (GGPAA), órgão colegiado de caráter deliberativo vinculado ao Ministério de Desenvolvimento Social e Combate à Fome, tem como objetivos orientar e acompanhar a implementação do PAA.

É relevante considerar toda a potencialidade do PAA, em proporcionar qualidade de vida para os agricultores fornecedores de alimentos, garantindo assim segurança alimentar para os recebedores. O programa estimula o consumo de alimentos regionais, agroecológicos e orgânicos, preservando muitos costumes, hábitos e culturas que vinham sendo esquecidos ao longo das gerações (GRISA et al., 2011).

Schmitt (2005) ressaltou que o PAA cria as condições necessárias para que o Estado possa atuar no mercado de produtos agrícolas, exercendo não apenas um efeito regulador sobre os preços regionais, mas dando um tratamento diferenciado para os agricultores familiares, buscando reforçar sua autonomia em relação aos chamados “atravessadores" e fortalecendo sua posição frente aos diferentes agentes de mercado.

É significativo evidenciar a multifuncionalidade do PAA, de ser canal de comercialização para agricultores familiares, propiciando o acesso à mercados institucionais e assegurar o fornecimento de alimentos saudáveis, garantindo segurança alimentar para pessoas em estado de vulnerabilidade alimentar e social, através das redes socioassistenciais. Por fim, sendo o principal agente para promover o diálogo entre instituições de esferas federal, estadual e municipal, ambas firmadas em um só propósito que é combater diretamente a insegurança alimentar e nutricional.

O programa se divide em seis modalidades diferentes, compra com doação simultânea, formação de estoques pela agricultura familiar, compra direta da agricultura familiar, incentivo à produção e incentivo de leite - PAA leite, compra institucional, aquisição de sementes. Cada modalidade tem alcance diferenciado de dois públicos beneficiários: os fornecedores e os consumidores de alimentos. Os fornecedores são agricultores familiares que através de um convênio firmado com o governo passa a fornecer alimentos. Os consumidores são instituições das redes socioassistenciais.

A modalidade compra com doação simultânea é a mais praticada no estado do Amazonas, tendo em vista que, consiste na compra de produtos que simultaneamente são 
CARNEIRO, J, P. R; PEREIRA, C. F; SANTIAGO, J. L; FRAXE, T. J. P.

doados a instituições socioassistenciais, suprindo a demanda local e promovendo o Direito Humano à Alimentação Adequada. A finalidade é incentivar a agricultura familiar local e promover a segurança alimentar e nutricional de indivíduos que se encontram em vulnerabilidade social e em situação de risco alimentar.

Neste sentido, é relevante apontar a dinâmica dos limites entre as modalidades, somando todas, exceto o PAA leite que não é executado no Amazonas, o limite pode chegar até $\mathrm{R} \$ 28.400,00$ (vinte e oito mil e quatrocentos reais) por unidade familiar/ano, podendo potencializar sua renda participando também em outras políticas públicas de fomento da agricultura familiar como o PNAE (Programa Nacional de Alimentação Escolar) e PREME (Programa de Regionalização da Merenda Escolar). Portanto, o mercado institucional é uma estratégia fundamental para viabilização do processo de comercialização rural no Amazonas, proporcionando o desenvolvimento econômico e estimulando a soberania alimentar.

\section{PARTICULARIDADES DO PAA NA ASSOCIAÇÃO DOS PRODUTORES RURAIS DA COMUNIDADE ESPERANÇA (ASPRONES).}

Agricultura familiar não é propriamente um termo novo, mas seu uso recente, com ampla penetração nos meios acadêmicos, nas políticas de governo e nos movimentos sociais, adquire novas significações. Quando o poder público implanta uma política federal voltada para este segmento, o Programa de Fortalecimento da Agricultura Familiar - PRONAF (BRASIL, 1996) ou quando cria a Lei 11.326/2006, a primeira a fixar diretrizes para o setor (BRASIL, 2006), a opção adotada para delimitar o público foi o uso "operacional" do conceito, centrado na caracterização geral de um grupo social bastante heterogêneo. Já no meio acadêmico, encontramos diversas reflexões sobre o conceito de agricultura familiar, propondo um tratamento mais analítico e menos operacional do termo.

No Estado do Amazonas a agricultura familiar apresenta especificidades e dimensões bem diferentes do restante do país. Em cada município sobressaem peculiaridades, dependendo da época do ano. A agricultura familiar ocupava mais de $91 \%$ dos estabelecimentos e 40,64\% das terras (MENEGHETTI e SOUZA, 2015). Em Rio Preto da Eva, assim como na maioria dos municípios do Amazonas a agricultura familiar é predominante. 
CARNEIRO, J, P. R; PEREIRA, C. F; SANTIAGO, J. L; FRAXE, T. J. P.

O município Rio Preto da Eva está situado na $7^{\text {a }}$ Sub-Região - Região do Rio Negro - Solimões, faz limite com os municípios de Itapiranga, Itacoatiara, Manaus e Presidente Figueiredo, sua área territorial é de 5.815,622 Km² (IBGE, 2018). É formado por aproximadamente 57 comunidades rurais (IDAM, 2010), sendo que a população se concentra em maior parte em área rural (52\%), fazendo com que o município seja essencialmente rural (VEIGA, 2002). Destaca-se no setor de fruticultura, que vem sendo evidenciado por sua grande diversidade e potencialidade de mercado, principalmente por conta de frutas de grande importância comercial como a banana, o coco e a laranja (OLIVEIRA et al., 2017).

Segundo dados da Conab (2018), os agricultores familiares do município de Rio Preto da Eva participam do PAA, desde 2010. Este município é o segundo no ranking dos municípios que mais possuem contratos com a CONAB, ficando atrás somente de Manacapuru. No ano de 2016, forneceu mais de 500 mil reais em produtos oriundos da agricultura familiar, fazendo com que se tornasse um dos principais produtores alimentos comercializados com a CONAB no Amazonas. Isso se dá através da contribuição das organizações produtivas, como exemplo, a Associação do Produtores Rurais da Comunidade Nova Esperança (ASPRONES), que foi responsável por mais de $50 \%$ do valor comercializado.

A ASPRONES conta aproximadamente com 65 agricultores familiares associados, dos quais cerca de 50 agricultores familiares participam ativamente das atividades da associação. As principais atividades são: compra coletiva de insumos para melhores condições de plantio; capacitação e oficinas sobre assuntos relacionados ao público alvo; parcerias com instituições governamentais e privadas com fim de proporcionar aos associados melhores oportunidades; planejamento, controle e gestão programas de comercialização rural através das compras institucionais.

Uma das mais importantes atividades da associação é a organização produtiva no acesso ao PAA, na modalidade Compra Doação Simultânea (CDS), cerca de 60 agricultores familiares participam. Onde é disponibilizado um valor de até 4.800/ano por família de agricultores familiares. A proposta é elaborada pela equipe administrativa da ASPRONES, que por sua vez passa somente a demanda documental para os associados, a responsabilidade de gestão, execução do programa, coleta e logística dos alimentos é de exclusividade de associação, para o agricultor familiar associado consiste somente a atribuição de plantar, colher e entregar sua produção na porta de sua casa. 
CARNEIRO, J, P. R; PEREIRA, C. F; SANTIAGO, J. L; FRAXE, T. J. P.

A associação dispõe de um caminhão baú, galpão para armazenamento da produção, uma equipe administrativa que absorve toda questão burocrática. Conforme os resultados do presente estudo, $100 \%$ dos agricultores familiares fornecedores entregam seus produtos por meio de estrada e utilizando o caminhão próprio da associação. Dessa forma, nota-se a grande vantagem de um grupo organizado que trabalha com uma configuração diferenciada no acesso a políticas públicas de comercialização rural, constata-se que a única e exclusiva preocupação dos agricultores familiares é aplicar todo seu tempo na sua produção.

Esse sistema de trabalho adotado pela ASPRONES, faz com que o acesso a modalidade do PAA se desenvolva de maneira crucial para o benefício e viabilização da comercialização rural, sobretudo, no que se refere a compras institucionais. Os principais produtos comercializados foram, banana, coco, laranja, cupuaçu, abacaxi, mamão, pimenta e mandioca. Vale ressaltar, que a banana é o principal produto negociado com o mercado institucional.

No início do ano de 2018, o PAA sofreu um grande corte no repasse de recurso para região Norte, em especial o Estado do Amazonas, essa severa diminuição trouxe inúmeros prejuízos aos associados, fazendo com que grande parte da produção dos agricultores familiares fosse vendida para atravessadores com preço muito abaixo do mercado, e no caso mais grave, se perdesse por falta de venda. No entanto, ao fim de 2018 surgiu novas demandas por parte da CONAB, trazendo assim, algumas expectativas de retomada de contrato com a associação, espera-se que ao início de 2019 a parceria se reestabeleça e que os agricultores sejam beneficiados, melhorando assim a sua qualidade de vida.

\section{SUSTENTABILIDADE DA AGRICULTURA FAMILIAR}

Refletir sobre a sustentabilidade da agricultura familiar, em uma abordagem da segurança alimentar, ajuda a amadurecer uma identidade compartilhada com os sujeitos sociais, mas respeitando a diversidade de opiniões e perspectivas sobre a apropriação do termo. Quando se analisa a sustentabilidade dos agricultores familiares associados a ASPRONES, a partir dos indicadores sociais, econômico, segurança alimentar e ambiental, as maiores contribuições foram dos agricultores fornecedores com os indicadores renda, segurança alimentar, que no seu contexto geral sobressaem sobre os não-fornecedores. 
CARNEIRO, J, P. R; PEREIRA, C. F; SANTIAGO, J. L; FRAXE, T. J. P.

Os aspectos ambientais apresentam algumas dimensões importantes que demonstram especificidades de práticas sustentáveis nas propriedades rurais de ambos os grupos, todavia, a sustentabilidade é constituída por vários aspectos, nesse contexto é possível analisar a junção de todos dados apresentados para criar indicadores que avaliem o impacto do PAA na sustentabilidade da agricultura familiar.

Na dimensão social, nota-se que duas variáveis importantes se destacam pela igualitariedade dos resultados entre os agricultores (Tabela 1), ambos os grupos apresentam os mesmos percentuais na idade (61 a 70) 40\%, e pessoa por residência (1 a 2 pessoas) 70\%. Essa semelhança aponta uma característica dentro do meio rural preocupante, pois assinala indícios de uma descontinuidade da prática rural, especificamente na agricultura, a idade foi a que dentre todas predominou entre os entrevistados, assim como a quantidade de pessoa por.

Esses dois indicadores apresentam um reflexo do panorama geral da população rural, o envelhecimento é constante e a quantidade de pessoas é cada vez menor, comprometendo a sustentabilidade social entre a população rural, pois se não existe outra geração sendo preparada ou assumindo o lugar de quem a vida toda se dedicou ao trabalho no campo, presumisse que a sustentabilidade corre risco de não se perpetuar.

No entanto, analisando os indicadores no seu contexto geral percebe-se que quanto ao gênero, a quantidade de mulheres foi bastante expressiva em ambos os grupos, isso apresenta de forma clara o incentivo de política públicas voltadas para o seguimento, proporcionando o protagonismo da mulher no campo. Quanto ao tempo de moradia o que mais se destacou entre os entrevistados foi o tempo de 11 a 20 anos, os fornecedores apresentaram o percentual de $50 \%$, ou seja, a metade dos fornecedores residem nesse espaço de tempo. Referente ao nível de escolaridade entre os entrevistados, os fornecedores também levam uma pequena vantagem, com $50 \%$ de sua população com o ensino fundamental completo.

Tabela 1 - Indicadores de Sustentabilidade - dimensão social

\begin{tabular}{ccc}
\hline Variáveis & Fornecedores & Não Fornecedores \\
Gênero & Frequência relativa $(\boldsymbol{F r})$ & Frequência relativa $(\boldsymbol{F r})$ \\
Feminino & $30 \%$ & $40 \%$ \\
Masculino & $70 \%$ & $60 \%$ \\
Idade & &
\end{tabular}


CARNEIRO, J, P. R; PEREIRA, C. F; SANTIAGO, J. L; FRAXE, T. J. P.

61 a 70

Escolaridade

Fundamental Completo

Pessoa por residência

1 a 2 pessoas

Tempo de Moradia

11 a 20 anos
$40 \%$

$40 \%$

$50 \%$

$40 \%$

$70 \%$

$70 \%$

$60 \%$

$50 \%$

Fonte: Carneiro, J.P.R. (2018)

O perfil econômico dos entrevistados é bem diversificado, várias fontes de renda compõem a renda geral de ambos os grupos (Tabela 2), é por meio de seu detalhamento que poderemos compreender se a sustentabilidade econômica de fato é existente. Dentre os grupos entrevistados, os fornecedores se destacam na renda obtida através do extrativismo e na produção agrícola, em contrapartida, os não-fornecedores se destacam na produção agrícola comercializada para o comércio local no acesso a benefícios sociais, no contexto geral a renda não dispõe de muita disparidade.

No entanto, analisando o critério de renda por salários mínimos os fornecedores concentram-se entre três a seis salários e sete a dez salários com os maiores percentuais, muito embora tenham também uma porcentagem de $30 \%$ de seus membros que estão na faixa etária de um a dois salários mínimos. É notório que a concentração de renda dos não fornecedores permaneça na sua maioria entre um a dois salários mínimos, com isso entende-se que o maior valor, se tratando em salários mínimos é dos fornecedores.

O programa em questão se torna uma ferramenta de fomento na renda de seus participantes, gerando autonomia e qualidade de vida, dentre os dois grupos estudados, os que mais se acessam novas fontes de renda a exemplo do PAA, são os fornecedores, diante disso, a sustentabilidade econômica pode ser visualizada de forma nítida, proporcionando estabilidade financeira e independência do ponto de vista econômico.

Tradicionalmente os agricultores familiares têm uma renda sazonal. Os fornecedores têm uma renda mensal, acesso ao mercado e maior rendimento econômico. Isto contribuiu para que os fornecedores tenham uma renda maior.

Tabela 2 - Indicadores de Sustentabilidade - dimensão econômica

\begin{tabular}{lll}
\hline Variáveis & Fornecedores & Não Fornecedores \\
\hline
\end{tabular}


CARNEIRO, J, P. R; PEREIRA, C. F; SANTIAGO, J. L; FRAXE, T. J. P.

\begin{tabular}{ccc}
\hline Composição da Renda & Frequência relativa $(\boldsymbol{F r})$ & Frequência relativa $(\boldsymbol{F r})$ \\
Produção agrícola - PAA & $11 \%$ & $0 \%$ \\
Produção agrícola - Comércio & & \\
local & $66 \%$ & $75 \%$ \\
Extrativismo & $18 \%$ & $2 \%$ \\
Benefícios Sociais & $5 \%$ & $23 \%$ \\
Salário mínimo & & $70 \%$ \\
1 a 2 & $30 \%$ & $20 \%$ \\
3 a 6 & $50 \%$ & $10 \%$ \\
7 a 10 & $20 \%$ & \\
\hline
\end{tabular}

Fonte: Carneiro, J.P.R. (2018)

A segurança alimentar é um componente importante na análise da sustentabilidade, a forma de se alimentar pode revelar muito sobre determinado grupo. Os agricultores familiares entrevistados responderam através da EBIA, questões que tratam diretamente sobre a preocupação com a alimentação, o dinheiro, a alimentação saudável e variada, os tipos de alimentos e a falta de alimento.

$\mathrm{O}$ resultado da pesquisa revelou que $48 \%$ dos não-fornecedores encontram-se em situação de insegurança alimentar, somente $24 \%$ dos fornecedores também estão em situação de insegurança alimentar. Esse resultado demonstra que embora trabalhem produzindo alimentos, na sua grande maioria se alimentam de forma inadequada, analisando os alimentos consumidos através da QFA. Pode-se notar que os alimentos saudáveis em grande parte são consumidos pelos fornecedores, porém os nãofornecedores alimentam-se mais de produtos industrializados.

O consumo de alimentos saudáveis por parte dos fornecedores pode ter sofrido grande influência do PAA, pelo fato do programa abastecer instituições socioassistenciais em situação de insegurança alimentar.

Vale ressaltar, é que no processo de entrevista com os agricultores fornecedores alguns afirmaram que entendem o principal objetivo da modalidade que participam, compra com doação simultânea, isso fez com que muitos mudassem alguns hábitos alimentares. Comparando os resultados dos dois grupos, afirma-se que os fornecedores é o grupo que desenvolve mais a sustentabilidade, do ponto de vista da segurança alimentar. 
CARNEIRO, J, P. R; PEREIRA, C. F; SANTIAGO, J. L; FRAXE, T. J. P.

Tabela 3 - Indicadores de Sustentabilidade - dimensão segurança alimentar

\begin{tabular}{ccc}
\hline Variáveis & Fornecedores & Não Fornecedores \\
\hline Segurança Alimentar & Frequência relativa $(\boldsymbol{F r})$ & Frequência relativa $(\boldsymbol{F r})$ \\
Sim & $24 \%$ & $48 \%$ \\
Não & $76 \%$ & $52 \%$ \\
\hline
\end{tabular}

Fonte: Carneiro, J.P.R. (2018)

Em relação a dimensão ambiental, e alguns aspectos foram levantados como podemos observar na tabela 4, por exemplo, “consome tudo que produzido aqui?”, todos entrevistados de ambos os grupos responderam que sim. A questão sobre aproveitamento dos resíduos sólidos foi algo surpreendente, apenas um agricultor familiar do grupo dos fornecedores afirmou que aproveita o resíduo sólido gerando, índice muito abaixo, tendo em vista que na maioria das vezes cerca de $80 \%$ do resíduo gerando pode ser reciclado.

Quanto ao sentimento de pertencimento e a relação com o meio ambiente, todos os entrevistados afirmaram que essa relação é excelente, se sentem satisfeitos com o lugar e tudo que é proporcionado, no que se refere aos recursos naturais e sua finitude, os fornecedores em sua totalidade afirmaram que são finitos, e que necessário o uso consciente, no grupo dos não fornecedores $90 \%$ acreditam ser finitos os recursos naturais disponibilizados.

A agricultura que vem sendo praticada pelos fornecedores é um sistema de produção sustentável. Constatou-se que somente $10 \%$ dos fornecedores utilizou mais de $20 \%$ de sua área total. No uso do agrotóxico, todos fornecedores entrevistados afirmaram não usar nenhum tipo de agrotóxico nas suas plantações, porém, $20 \%$ dos nãofornecedores fazem uso de algum tipo de agrotóxico. Finalizando com quem faz mais queimadas, os não-fornecedores praticam de forma intensa essa prática. Analisando os resultados obtidos da dimensão ambiental, os fornecedores possuem práticas ambientais mais sustentáveis, comparando aos não-fornecedores.

Tabela 4 - Indicadores de Sustentabilidade - dimensão ambiental

\begin{tabular}{ccc}
\hline Variáveis & Fornecedores & Não Fornecedores \\
\hline Ambiental & Frequência relativa $($ Fr $)$ & Frequência relativa $(F r)$
\end{tabular}


CARNEIRO, J, P. R; PEREIRA, C. F; SANTIAGO, J. L; FRAXE, T. J. P.

\begin{tabular}{|c|c|c|}
\hline Recursos naturais são finitos & $14 \%$ & $13 \%$ \\
\hline $\begin{array}{l}\text { Ótima relação com o meio } \\
\text { ambiente }\end{array}$ & $14 \%$ & $14 \%$ \\
\hline $\begin{array}{c}\text { Sentimento de pertencimento do } \\
\text { lugar }\end{array}$ & $14 \%$ & $14 \%$ \\
\hline Aproveita os resíduos Sólidos & $1 \%$ & $0 \%$ \\
\hline Não utiliza agrotóxico & $14 \%$ & $11 \%$ \\
\hline Faz queimadas & $4 \%$ & $8 \%$ \\
\hline Consome tudo que é produzido & $14 \%$ & $14 \%$ \\
\hline $\begin{array}{c}\text { Respeita a Legislação } \\
\text { Ambiental }\end{array}$ & $13 \%$ & $13 \%$ \\
\hline Coleta de água - Poços & $9 \%$ & $14 \%$ \\
\hline
\end{tabular}

Fonte: Carneiro, J.P.R. (2018)

O resultado permite concluir que o impacto do PAA na agricultura familiar é sustentável, embora em alguns aspectos o comparativo entre os grupos se assemelha, porém, a hegemonia é dos fornecedores. Sendo assim é plausível afirmar que o PAA na organização social, renda, segurança alimentar e ambiental foi de vital importância para a construção da sustentabilidade. Portanto os efeitos do PAA na vida dos agricultores familiares participantes trouxeram impactos positivos, de forma que esses impactos se demonstraram na maneira de organização do grupo, na autonomia financeira, no consumo de alimentos saudáveis e nas práticas sustentáveis.

\section{CONSIDERAÇÕES FINAIS}

Os resultados desta pesquisa elucidaram que os aspectos avaliados ao longo do trabalho, como indicadores socioeconômicos traça um perfil mais específico dos agricultores familiares associados a ASPRONES, realizado através de um comparativo entre os agricultores fornecedores e não-fornecedores. Os agricultores fornecedores estão bem mais estruturados do ponto de vista social e econômico. Tendo em vista que no seu contexto geral são bem semelhantes, porém, quando se avalia de forma mais detalhada encontramos uma pequena diferença, se tornando assim, um ponto crucial na avaliação dos grupos. 
O consumo alimentar entre os grupos apresentou de modo sucinto, que o PAA tende a ser um forte incentivador e promotor de segurança alimentar e nutricional dos agricultores familiares fornecedores, contribuindo para mitigação da insegurança alimentar. Com efeito, a segurança alimentar mostrou-se evidente através dos resultados, que além de tudo, notou-se que os fornecedores se alimentam de maneira mais saudáveis, se valendo do fato que estão produzindo de maneira mais segura, pois, isso foi demonstrado claramente na pesquisa, que os fornecedores em sua totalidade não fazem uso de agrotóxicos em suas plantações.

As práticas ambientais também mostram que os agricultores familiares fornecedores, evidenciam um perfil mais sustentável no que se refere ao trato com meio ambiente. Alguns pontos são importantes destacar, o uso da área produtiva apontou que os fornecedores possuem as maiores áreas consolidadas, porém dentro das especificações da legislação ambientais. Nesse sentido, o modo de vida dos agricultores familiares, promove e corrobora com a sustentabilidade ambiental, em suas diferentes facetas.

Alguns fatores contribuíram de forma direta para o resultado da pesquisa, um deles foi a organização social dos agricultores familiares associados a ASPRONES, a associação dispõe de uma característica peculiar, trabalha de forma intensa para que seus associados procurem somente priorizar a sua produção, pois assuntos burocráticos que são necessários para o acesso ao PAA é realizado exclusivamente pela associação, isso torna crucial o processo produtivo dos agricultores familiares, fazendo que o aumento da produção seja intensificado, gerando assim, mais renda e qualidade de vida, sem deixar as práticas sustentáveis de lado.

Portanto, os resultados demonstraram que a PAA contribui para sustentabilidade da agricultura familiar, à medida que a ASPRONES vai se consolidando no acesso ao mercado institucional, existe uma tendência de incorporação dos agricultores nãofornecedores e levará ao equilíbrio entre as diferentes dimensões da sustentabilidade.

Este trabalho contribui na avaliação dos impactos do PAA na agricultura familiar. Concluiu-se que o PAA tem a função de atuar como promotor de sustentabilidade nas suas mais diversas formas de transversalidade, entretanto, aponta para necessidades de trabalhos futuros, aprofundando-se os indicadores de que envolvam o capital social na sustentabilidade da agricultura familiar.

Os resultados apontam a eficiência do PAA no seu contexto mais abrangente, abarcando desde suas origens, execuções, modalidades chegando até seu ápice, demonstrando como pode ser também um canal para construir a sustentabilidade. Esse 
entendimento tornou-se essencial para traçar novos caminhos a partir dos resultados da pesquisa, no qual evidencia aspectos importantes da sustentabilidade.

Compreender a sustentabilidade através da ótica das políticas públicas, sobretudo aquelas que promovem a importância da agricultura familiar, foi de fato o grande desafio desta dissertação. É importante ressaltar que o fortalecimento de políticas voltadas para a agricultura familiar mostra-se como estratégia de sustentabilidade, assim como a inserção da agricultura familiar como estratégia de desenvolvimento rural.

\section{REFERÊNCIAS}

BECKER C, SACCO DOS ANJOS F. Segurança alimentar e desenvolvimento rural: limites e possibilidades do Programa de Aquisição de Alimentos da agricultura familiar, em municípios do sul gaúcho. Revista Seg. Alim Nutr. 2010; p.61-72.

BRASIL. Decreto $n^{\circ} 1.946$, de 28 de junho de 1996. Cria o Programa Nacional de Fortalecimento da Agricultura Familiar - PRONAF, e dá outras providências. Diário Oficial da União. Brasília, DF, 28 jun. 1996. Disponível em: < http://www.planalto.gov.br/ccivil_03/decreto/D1946.htm >: 23 jan. 2018.

BRASIL. Lei no 11.326, de 24 de julho de 2006, estabelece as diretrizes para a formulação da Política Nacional da Agricultura Familiar e Empreendimentos Familiares Rurais. Brasília, DF, 24 jul. 2006. Disponível em: < http://www.planalto.gov.br/ccivil_03/_Ato2004-2006/2006/Lei/L11326.htm>. Acesso em: 23 jan. 2018.

BRASIL. Decreto $n^{\circ} 7775$, de 4 de julho de 2012. Regulamenta o art. 19 da Lei no 10.696, de 2 de julho de 2003, que institui o Programa de Aquisição de Alimentos, e o Capítulo III da Lei no 12.512, de 14 de outubro de 2011, e dá outras providências. Diário Oficial da União. Brasília, DF, 5 jul. 2012. Disponível em: <http://www.planalto.gov.br/ccivil_03/_ato2011-2014/2012/Decreto/D7775.htm>.

Acesso em: 23 jan. 2018.

COMPANHIA NACIONAL DE ABASTECIMENTO. Compêndio de Estudos Conab / Companhia Nacional de Abastecimento. - V. 1 (2017- ). - Brasília: Conab, 2017. GRISA, C. et al. Contribuições do Programa de Aquisição de Alimentos à segurança alimentar e nutricional e à criação de mercados para a agricultura familiar. Agriculturas, Rio de Janeiro, v. 8, n. 3, p. 34-41, set. 2011.

IBGE - INSTITUTO BRASILEIRO DE GEOGRAFIA E ESTATÍSTICA. Pesquisa Nacional por Amostra de Domicílios. PNAD, 2013. Disponível em: www.ibge.gov.br/home/estatistica/populacao/segurança_alimentar_2013. Acesso em: 23 jan. 2018.

MENEGHETTI, G. A.; SOUZA, S. R. A agricultura familiar do Amazonas: conceitos, caracterização e desenvolvimento. Revista Terceira Margem Amazônia. v.1 n.5, p. 5974. 2015. 
MÜLLER, A.L; FIALHO, A.V, SCHNEIDER, S. A. Inovação institucional e a atuação dos atores locais na implementação do Programa de Aquisição de Alimentos no Rio Grande do Sul. Sociedade e Desenvolvimento Rural. 2007;1(1):1-21.

OLIVEIRA, E.C; NASCIMENTO, J.D.R; LIMA, I.A.S; SILVA, Y.Z. O setor de fruticultura do estado do Amazonas: análise e desafios para o desenvolvimento regional. Disponível em: <https://even3storage.blob.core.windows.net/processos/e77397e89efc45ee8c62.pdf > Acesso em 27 de fevereiro de 2019.

SOARES, Panmela; MARTINELLI, Suellen Secchi; MELGAREJO, Leonardo; CAVALLI, Suzi Barletto. Fornecimento de alimentos da agricultura familiar para a alimentação escolar: o exemplo do Programa de Aquisição de Alimentos. Segurança Alimentar e Nutricional, Campinas, 20(1): 41-51, 2013.

VEIGA, José Eli da. Cidades Imaginárias - o Brasil é menos urbano do que se calcula. Campinas: Editora da Unicamp, 2005.

YAZBEK, M. C. O Programa Fome Zero no Contexto das Políticas Sociais Brasileiras. São Paulo em Perspectiva, São Paulo, v. 18, n. 2 Jun 2004, p. 104-112, 2004.

YIN, R. K. Estudo de caso: planejamento e métodos. Trad. Daniel Grassi. Porto Alegre: Bookman, 2001. 